\title{
Constructos teóricos para abordar, de un modo investigativo, problemas entre marketing, producción y logística en las empresas colombianas
}

\author{
Theoretical constructs to address, via research, problems among marketing, production, and logistics in
} colombian companies

Construits théoriques pour aborder, á mode de recherche, des problèmes entre le marketing, la production et la logistique dans les entreprises colombiennes

\begin{abstract}
Alexander Varón Sandoval
Docente Departamento de Administración y Organizaciones, Área de Mercadeo, Maestría en Ciencias de la Organización (c), Maestría en Ciencias Biomédicas (c), Universidad del Valle, Cali - Colombia. Máster en Marketing, Universidad Autónoma de Madrid - España. Especialista en Gerencia de Producción con concentración en Logística Empresarial - Universidad Icesi. E-mail: Alexander.varon@correounivalle.edu.co

Artículo de investigación científica y tecnológica según clasificación COLCIENCIAS

Recepción: 14/02/2013

Corrección: $14 / 04 / 2013$

Aprobación: 05/06/2013
\end{abstract}

\section{Resumen}

Este artículo propone un modelo de constructo teórico, a partir de una investigación cualitativa, por medio de cuestionario con preguntas abiertas y una revisión teórica. La población objeto de estudio estuvo representada por los colaboradores de los departamentos de marketing, producción y logística, de algunas empresas de manufactura en Colombia indistintamente seleccionados, entre los años 2006 y 2007 . El objetivo fue generar constructos teóricos que sirvieran de base para la investigación de los conflictos entre marketing, producción y logística, dentro de las empresas colombianas, justificado en la presencia del conflicto, en ese momento poco estudiado, entre estas áreas de la organización. En la metodología, se establecieron varias etapas, entre ellas: a) la recopilación de información pertinente, b) encuestas a personas que habían trabajado o estuvieran trabajando en alguna de las áreas de interés, c) determinación de un marco teórico y conceptual, a partir de la revisión de autores especializados en este tema.

Palabras clave: conflictos interdepartamentales, marketing, producción, logística.

\begin{abstract}
This article proposes a theoretical construct model, from an open-questions instrument in a qualitative research methodology and theoretical revision. This research was conducted among employees of the departments of marketing, production and logistics of some manufacturing companies in Colombia, between 2006 and 2007. The research objective was to generate theoretical constructs to investigate conflicts among marketing, production and logistics, in Colombian companies due the presence of conflicts among those areas and a lack of specific research at that time. In the methodology, several stages were established; these include: a) gatheringof relevant information, b) surveys of people who have worked or work in any area of interest, c) determination of the theoretical and conceptual framework from the perspective of authors specialized in this issue.

Keywords: inter-departmental conflicts, marketing, production, logistics.

\section{Résumée}

Cet article propose un modèle de construit théorique à partir d’une recherche qualitative, par le biais d'un questionnaire avec des questions ouvertes et une révision théorique. La population objet d’étude a été représentée par les collaborateurs des départements de marketing, de production et de logistique de quelques entreprises de manufacture en Colombie, indistinctement sélectionnées entre 2006 et 2007. Ĺobjectif a été celui de créer des construits théoriques capables de servir comme base pour la recherche des conflits entre marketing, production et logistique, à l`intérieur des entreprises colombiennes, ceci est justifié par la présence du conflit, à ce moment peut étudié, entre ces aires de l'organisation. Dans la méthodologie on a établi plusieurs étapes, parmi elles : a) la recompilation d`information pertinente, b) enquêtes aux personnes qui avaient travaillé ou travaillaient en ce moment dans une des aires d`intérêt, c) détermination d’un cadre théorique et conceptuel, à partir de la révision des auteurs spécialisés sur le sujet.
\end{abstract}




\section{Introducción}

A través de la historia, se ha observado que las empresas en cada fase de su desempeño - desde la orientación hacia el producto hasta la orientación hacia el mercado- han rotado sus puntos clave por cada una de las diferentes áreas que las componen. De los silos funcionales llegaron al logro de la premisa máxima: la satisfacción del consumidor. De ahí que, hoy en día, los conceptos de cadena de valor, productividad, calidad y satisfacción del consumidor representen la mayor preocupación de las empresas.

Es un hecho que dentro de cada una de las áreas de la compañía se presentan conflictos. A pesar de que se hacen algunos esfuerzos para lograr una buena administración, cada vez son más grandes y de mayor calibre. Las áreas más afectadas son las de logística, producción y marketing, precisamente, en las que se enfoca el presente estudio. De igual manera que se han generado modelos de negociación productivos para las empresas en las áreas de marketing y ventas, es posible proponer un modelo que propicie la interacción sana y conjunta de estas tres áreas que viven en conflicto permanente: logística, producción y marketing.

En este artículo, se presenta el desarrollo de la Tesina de Grado desarrollada entre los años 2006 y 2007, como requisito para optar al título de Máster en Marketing, de la Universidad Autónoma de Madrid, España. Esta investigación se realizó con el apoyo del Programa de Becas de Alto Nivel (ALBAN) de la Unión Europea. Lo que motivó al tesista a abordar este tema fue la urgencia de reducir los conflictos de indole negativa que tienen las empresas en Colombia y sentar bases para investigar y aplicar modelos de negociación entre sus departamentos de marketing, producción y logística.

\section{Marco teórico}

\subsection{Generación de conflictos}

No existe un criterio único en cuanto a la definición precisa de conflicto, pero, de acuerdo con Xie (1998), en un contexto interfuncional o interdepartamental, se puede definir como "las diferencias percibidas en cuanto a objetivos e ideologías a través de áreas interdependientes e interactivas". Thomas (1992), por su parte, dice que es un proceso que comienza cuando una de las partes involucradas percibe que está afectando o viéndose afectada negativamente por algo de lo cual es responsable otra de las partes. Dyer \& Song (1997) sostienen que el conflicto es un desacuerdo relacionado con la tarea, pues las partes perciben que las metas u objetivos de otras de las partes son incompatibles con los propios.

En otros estudios se han involucrado los factores de la teoría de la identidad social, la cual puede ser aplicada directamente en cualquier grupo. Esta teoría plantea que el sentido de pertenencia hacia un grupo hace que sus miembros piensen de manera instantánea, que el esfuerzo -por los valores u objetivos perseguidos- de los otros grupos es menor (Brewer, 1986). Por tanto, se concibe como una fuente de conflicto y, como tal, puede ser alimentada, si no se tiene una percepción clara de los objetivos organizacionales, 0 si existen unas fronteras demasiado definidas entre unos y otros departamentos.

El conflicto, como hemos visto, es un desacuerdo ya sea entre intereses, ideas, o ambos, cuyos objetivos organizacionales difieren de un actor a otro (Pondy, 1966). Históricamente, se ha considerado el conflicto como indeseable y hay quienes piensan que debe ser evitado (Higgins, 1991; Thompson, 1960). Incluso algunos directivos ven con mala cara, a aquellas personas que con frecuencia están involucradas en conflictos -sea cual sea la naturaleza de los mismos-y los etiquetan como "los problemáticos" o "las manzanas podridas" (Whethen \& Cameron, 1995).

Respecto a este particular, se puede decir que el conflicto puede afectar el desempeño, aunque existen estudios que demuestran lo contrario. Por tanto, el conflicto, dependiendo de su naturaleza y momento en que se genera, puede tener connotaciones tanto negativas como positivas. Brown (1983), Schwenk \& Cosier (1993) encontraron que los conflictos eran destructivos y que disminuían el desempeño de los grupos. Pero investigadores como Baron (1991), Bourgeois (1985) y Putnam (1994) opinan que el conflicto es beneficioso y ayuda a incrementar el desempeño grupal. Lo mismo piensan Thamhain y Wilemon (1975), quienes trataron de explicar esta ambigüedad a través de diferentes estudios de ingeniería administrativa. Ellos demostraron claramente que el grado de beneficio o daño depende directamente de los líderes. Dejaron claro que si los líderes eran asertivos, las consecuencias de los conflictos eran benéficas y por tanto, se minimizaban los perjuicios del mismo.

Con el fin de analizar mejor el contexto del conflicto, es pertinente tener en cuenta lo siguiente: a) saber que sus efectos están relacionados con los siguientes factores: nivel de incertidumbre, nivel de variabilidad de la labor, cantidad de información proveniente de las partes involucradas, entre otros. b) Establecer las relaciones interfuncionales existentes, porque las metas u objetivos propuestos se verán afectados. c) Revisar analíticamente los diferentes tipos de resolución de conflictos para escoger el método o métodos adecuados. d) Determinar si existen diferencias culturales entre cada una de las diferentes áreas involucradas en el conflicto. e) Conocer la existencia de factores que no sólo van a propiciar el conflicto, sino que lo van a promover. Por estas razones, se deben considerar las diferencias en cuanto a las metas y los valores de cada área, la conexión que existe entre la emoción y la tarea que se desarrolla y la disponibilidad de recursos para manejar el conflicto, pues esto, además de consumir tiempo, consume recursos adicionales.

Otros estudiosos como Esquivel (1997), plantean que existen dos clases de conflicto: el tipo $\mathrm{C}$ y el tipo A. El conflicto tipo $C$ es aquel, cuyos protagonistas se enfocan en los aspectos sustantivos u objetivos de las diferencias de opinión. Las interacciones y discusiones se fundamentan en una comunicación honesta y abierta, basada en las diferentes habilidades y destrezas de los miembros del equipo; como se puede observar, este tipo de conflicto tiende a mejorar la efectividad de los resultados del equipo (Amason, 1995). 
Mientras este tipo de conflicto fomenta la creatividad, el uso de las habilidades y destrezas y el ejercicio de una comunicación abierta y honesta, el conflicto tipo A hace todo lo contrario: los hechos dejan de ser objetivos y se trasladan al plano personal y emocional, pues se le agrega el componente de la subjetividad. Por supuesto, esto trae malas consecuencias como la disminución en la efectividad del equipo, limitación de la creatividad, incremento de la hostilidad, enojo y pérdida de la confianza. De este modo, el conflicto, como tal, presenta tres características generales: la interacción, la interdependencia y la incompatibilidad de objetivos o metas (Xie, 1998).

\subsubsection{Los conflictos desde el departamento de marketing hacia otros departamentos en general}

Para comprender la naturaleza del conflicto de las áreas de nuestro interés con el área de marketing, es necesario mencionar algunas áreas que entran en conflicto con ésta, entre las cuales están: comercial y ventas, logística de distribución, producción. Se puede decir que, por un lado, está la función de marketing estudiando al consumidor, analizando los beneficios que deben respaldar su oferta y emitiendo los programas de comunicación; por el otro, está ventas que actúa sobre los decisores de compra.

En el medio, está el Trade Marketing que surge como respuesta a los conflictos que se generan entre estas dos áreas. Es una alianza estratégica per se entre productores y distribuidores para el desarrollo de acciones conjuntas (Sampietro, 2006). En otras palabras, es el eslabón estratégico entre marketing y ventas. Con esta función, se han logrado dos tipos de ventajas competitivas: a) en la operación: mayor capacidad y rapidez de respuesta al cliente, mediante un área de servicios centrada en la distribución y en el cliente; b) en lo estratégico: una visión orientada a largo plazo, enfocada más en la marca que en el cliente.

\subsubsection{Los conflictos entre marketing y los departa- mentos de producción y logística}

Shapiro (1977) afirmó que los departamentos de producción (back-office department) y marketing (front-office department) podían coexistir, aunque también aclaró sus diferencias. En efecto, indicó que los departamentos back-office usualmente propenden por la reducción de costos, mediante la eficiencia en la producción, mientras que los departamentos front-office lo hacen por maximizar el beneficio y se adaptan a los requerimientos del consumidor. En este sentido, Shapiro vislumbraba uno de los mayores problemas entre estos dos departamentos: la diferencia de objetivos y metas. Asimismo, lo planteó Nauta (2001): “Las percepciones de los objetivos, entre los departamentos, pueden ser una mayor fuente de problemas en la coordinación inter-departamental que un conflicto entre departamentos, debido a que dichas percepciones implican la creencia de que los objetivos de los grupos respectivos son incompatibles".

Varias investigaciones (Bazaraa y Jarvis, 1977; Burns y Stalker, 1966; Dahler-Larsen, 1998; Galbraith, 1973; Mintzberg, 1979; Ouchi, 1980; Walton y Lawrence, 1985, citados por Nauta, 2001), también, han concluido que las fuentes de conflictos, generados entre marketing y producción, son los objetivos y las metas. Precisamente, la de Nauta (2001) es la que reúne los esfuerzos de la gran mayoría. En ella, se identificaron, como metas propias de estos departamentos, las siguientes: eficiencia, calidad, rapidez en la entrega, confiabilidad en la entrega, flexibilidad y servicio al cliente. Eficiencia tiene que ver con la minimización de costos; calidad, con productos buenos; confiabilidad, con entregas a tiempo; flexibilidad, con el ofrecimiento de una variedad de productos, cantidades y tiempos de respuesta; servicio al cliente, con el ofrecimiento -al consumidor- de servicios e información sobre el producto, metas que entran en conflicto cuando se quieren alcanzar independientemente de los otros departamentos con que se interactúan.

Muchos estudios han encontrado que la calidad de la interacción entre estos departamentos, estaba estrechamente relacionada con el desempeño corporativo (Lawrence y Lorsch, 1967; Hayes y Wheelwright, 1984; Powers, 1988; Crittenden, 1992; Crittenden, 1993; Miller y Roth, 1994; Prabhaker, 1995; Kamarkar, 1996). El desempeño corporativo hace referencia a los objetivos estratégicos de la compañía y cómo son interpretados dentro de las tácticas operacionales inherentes a los departamentos o áreas funcionales. Por ejemplo, el objetivo principal del departamento de producción es la eficiencia enfocada hacia la producción. Para lograr la eficiencia en la producción, es necesario tener lotes productivos más largos, lo cual genera economías de escala y curvas de aprendizaje, sistemas de calidad firmes y sencillez en los procesos de producción. Todo esto implica una sobrecarga para el departamento de marketing, cuyos funcionarios no pueden ceder ante los requerimientos de los clientes, aunque probablemente, los pierdan en el mar de los competidores.

Si se quiere una eficiencia en marketing -adaptada a los requerimientos del consumidor- producción deberá hacer entregas en un tiempo menor al presupuestado, como también, cambios en el tipo y/o especificaciones del producto. Esto produciría lotes de producción más pequeños, exigiría modificaciones mayores en el último momento del plan maestro de producción y generaría una sobrecarga en dicho departamento, por ende, problemas asociados con los procesos productivos y la calidad del producto (Gráfico 1).

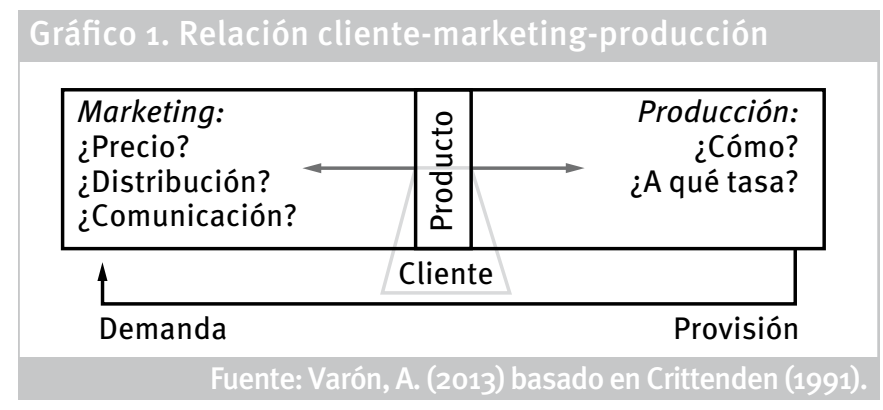

En otras palabras, si las decisiones en lo operativo son guiadas por objetivos propios de cada área, más que por los objetivos estratégicos, el resultado que se 
obtiene será inconsistente y contraproducente (Goldratt y Cox, 1993).

De acuerdo con la teoría de la identidad social, tener pertenencia por un grupo o departamento y no por otros, crea una percepción sesgada a favor del propio grupo o departamento y un rechazo hacia los demás (Brewer, 1986; Brewer y Schneider, 1990). Esta teoría propone que los individuos se definan a sí mismos con los términos del grupo del que son miembros, que busquen una identidad social positiva pero sin pensar que el grupo al que pertenecen es mejor que los demás. Si se hace la diferencia, los miembros de determinado grupo se verán como los buenos y a los otros, como los malos. Lamentablemente, existen estímulos que refuerzan este tipo de comportamientos. Por ejemplo, producción es usualmente recompensado por la eficiencia y calidad; logística, por la planificación y la entrega a tiempo, y marketing, por la flexibilidad y servicio al cliente. Estos estímulos, además de generar rechazos, pueden en cierta medida, dar a entender que se persiguen objetivos diferentes. De igual manera, características organizacionales, como el tipo de estrategia, estructura, cultura, sistema de producción, sistema de planificación, entre otros, representan la fuente que determina el grado de diferencia en objetivos. Otras fuentes de conflicto entre estas dos áreas incluyen la separación física de las dos funciones, la orientación individual, la diferencia en los requerimientos de información, las diferencias culturales, la ambigüedad de la estrategia corporativa, la presión por crecer rápidamente, los cambios ambientales, la proliferación de operaciones automatizadas, las restricciones de capital y el tamaño de la empresa.

En las Tablas 1 y 2, se recopilan los posibles puntos de conflicto entre marketing y producción.

\begin{tabular}{|c|c|c|}
\hline Punto de conflicto & Objetivo de marketing & $\begin{array}{l}\text { Objetivo de } \\
\text { producción }\end{array}$ \\
\hline \multicolumn{3}{|c|}{ Administración de la diversidad } \\
\hline $\begin{array}{l}\text { Ancho y largo de la } \\
\text { línea de producto }\end{array}$ & $\begin{array}{l}\text { Muchos y complejos } \\
\text { modelos }\end{array}$ & $\begin{array}{l}\text { Pocos y sencillos } \\
\text { modelos }\end{array}$ \\
\hline $\begin{array}{l}\text { Personalización del } \\
\text { producto }\end{array}$ & $\begin{array}{l}\text { Especificaciones del } \\
\text { cliente }\end{array}$ & Productos en "stock" \\
\hline $\begin{array}{l}\text { Cambios en la línea de } \\
\text { producto }\end{array}$ & $\begin{array}{l}\text { Cambios inmediatos } \\
\text { en la línea de } \\
\text { producto: alto riesgo }\end{array}$ & $\begin{array}{l}\text { Cambios planeados, } \\
\text { solos los necesarios: } \\
\text { bajo riesgo }\end{array}$ \\
\hline \multicolumn{3}{|c|}{ Administración de la conformidad } \\
\hline $\begin{array}{l}\text { Planificación de la } \\
\text { producción }\end{array}$ & Cambio constante & Inflexible \\
\hline $\begin{array}{l}\text { Planificación de la } \\
\text { capacidad-facilidad }\end{array}$ & $\begin{array}{l}\text { Aceptar todas las } \\
\text { órdenes }\end{array}$ & $\begin{array}{l}\text { Evaluación crítica de } \\
\text { las órdenes que se } \\
\text { ajustan }\end{array}$ \\
\hline \multicolumn{3}{|c|}{ Administración de la formalidad } \\
\hline Delivery & $\begin{array}{l}\text { Inmediato, grandes } \\
\text { inventarios }\end{array}$ & $\begin{array}{c}\text { Tan pronto como sea } \\
\text { posible, no inventarios }\end{array}$ \\
\hline Control de calidad & Altos estándares & Control razonable \\
\hline \multicolumn{3}{|c|}{ Fuente: Varón, A. (2013) basado en Crittenden (1991, p. 27). } \\
\hline
\end{tabular}

\begin{tabular}{|c|c|c|c|}
\hline \multirow{2}{*}{\multicolumn{2}{|c|}{ Área de problema }} & \multicolumn{2}{|c|}{ Comentarios típicos } \\
\hline & & De marketing & De producción \\
\hline 1 & $\begin{array}{l}\text { Planificación de la capacidad y } \\
\text { pronósticos de ventas a largo plazo }\end{array}$ & ¿Por qué no tenemos suficiente capacidad? & $\begin{array}{l}\text { ¿Por qué no tenemos pronósticos de ventas } \\
\text { acertados? }\end{array}$ \\
\hline 2 & $\begin{array}{l}\text { Programación de la producción y } \\
\text { pronósticos de ventas a corto plazo }\end{array}$ & $\begin{array}{l}\text { Necesitamos una respuesta más rápida, nuestros } \\
\text { tiempos de espera son ridículos }\end{array}$ & $\begin{array}{l}\text { Necesitamos compromisos reales de clientes y } \\
\text { pronósticos de ventas que no cambien como } \\
\text { cambia la dirección del viento }\end{array}$ \\
\hline 3 & Entrega y distribución física & $\begin{array}{l}\text { ¿Por qué no contamos nunca con la mercancía } \\
\text { correcta en el inventario? }\end{array}$ & No podemos mantener de todo en el inventario \\
\hline 4 & Aseguramiento de la calidad & $\begin{array}{l}\text { ¿Por qué no podemos tener una calidad razonable a } \\
\text { un costo razonable? }\end{array}$ & $\begin{array}{l}\text { ¿Por qué debemos siempre ofrecer opciones que } \\
\text { son de difícil producción y que ofrecen muy poca } \\
\text { utilidad al cliente? }\end{array}$ \\
\hline 5 & Amplitud de la línea de producto & Nuestros clientes demandan variedad & $\begin{array}{l}\text { La línea de producto es demasiado amplia - todo lo } \\
\text { que tenemos es para cortas y poco económicas } \\
\text { corridas de producción }\end{array}$ \\
\hline 6 & Control de costos & $\begin{array}{l}\text { Nuestros costos son tan altos que no somos } \\
\text { competitivos en el mercado }\end{array}$ & $\begin{array}{l}\text { No podemos proveer una entrega rápida, amplia } \\
\text { variedad, respuestas rápidas al cambio y alta } \\
\text { calidad a un bajo costo }\end{array}$ \\
\hline 7 & Introducción de nuevos productos & Los productos nuevos son nuestra sangre vital & $\begin{array}{l}\text { Cambios innecesarios en el diseño son } \\
\text { supremamente caros }\end{array}$ \\
\hline 8 & $\begin{array}{l}\text { Servicios adjuntos como soporte de } \\
\text { inventario de partes separadas, } \\
\text { instalación y reparación. }\end{array}$ & $\begin{array}{l}\text { Los costos de los servicios de campo son } \\
\text { demasiado altos }\end{array}$ & $\begin{array}{l}\text { Los productos están siendo utilizados en formas } \\
\text { para las que no han sido diseñados. }\end{array}$ \\
\hline
\end{tabular}

1. Shapiro enmarca estos conflictos desde otras dimensiones, estableciendo los típicos comentarios asociados con la presencia de conflictos entre las áreas de marketing y producción. 
Las causas básicas del conflicto entre estos dos departamentos aparecen en la Tabla 3.

\begin{tabular}{|c|c|c|c|}
\hline \multicolumn{2}{|c|}{$\begin{array}{l}\text { Evaluación y } \\
\text { recompensa }\end{array}$} & \multicolumn{2}{|c|}{$\begin{array}{l}\text { Las dos funciones son evaluadas en función de } \\
\text { criterios diferentes y reciben recompensa por } \\
\text { diferentes actividades. Como cada grupo le interesa } \\
\text { ser bien evaluado y recompensado, responde, de } \\
\text { acuerdo con lo que se le exige, protegiendo sus } \\
\text { propios intereses. }\end{array}$} \\
\hline \multicolumn{2}{|c|}{$\begin{array}{l}\text { Complejidad } \\
\text { inherente }\end{array}$} & \multicolumn{2}{|c|}{$\begin{array}{l}\text { Debido a que estas áreas representan el motor real } \\
\text { de la compañía, entonces, existe una inherente } \\
\text { complejidad tanto operacional como conceptual en } \\
\text { diferentes niveles internos de cada área. }\end{array}$} \\
\hline \multicolumn{2}{|c|}{$\begin{array}{l}\text { Orientación y } \\
\text { experiencia }\end{array}$} & \multicolumn{2}{|c|}{$\begin{array}{l}\text { El director de cada área está más preocupado por su } \\
\text { propia situación organizacional que la de los } \\
\text { demás. }\end{array}$} \\
\hline \multicolumn{2}{|c|}{$\begin{array}{l}\text { Diferencias } \\
\text { culturales }\end{array}$} & \multicolumn{2}{|c|}{$\begin{array}{l}\text { Tanto las tareas como las preocupaciones sociales } \\
\text { generan diferencias que serán fuente de conflicto. }\end{array}$} \\
\hline \multicolumn{4}{|r|}{ Fuente: Varón, A. (2013) } \\
\hline \multicolumn{4}{|c|}{$\begin{array}{l}\text { Dependiendo del mercado donde la organización } \\
\text { establece sus operaciones, existen dos tipos extremos } \\
\text { de estrategias organizacionales. Una de ellas es la de } \\
\text { bajo costo y la otra es la de competir con alto servicio al } \\
\text { cliente (Porter, 1980). El Gráfico } 2 \text { explica la relación entre } \\
\text { las características del mercado y el tipo de estrategia de } \\
\text { la empresa que correspondería a este mercado. }\end{array}$} \\
\hline \multicolumn{4}{|c|}{ Gráfico 2 Relación variedad vs. volumen } \\
\hline \multirow{3}{*}{$\begin{array}{l}+ \\
\frac{c}{\Phi} \\
\stackrel{c}{3} \\
\frac{0}{\circ} \\
-\end{array}$} & \multicolumn{2}{|c|}{$\begin{array}{c}\text { Estrategia de } \\
\text { bajo costo }\end{array}$} & \\
\hline & & & \begin{tabular}{|c|} 
Estrategia de \\
servicio al cliente \\
\end{tabular} \\
\hline & \multicolumn{3}{|c|}{ - Variedad + } \\
\hline \multicolumn{4}{|c|}{$\begin{array}{l}\text { Fuente: Varón, A. (2013) basado en Porter (1980), Shapiro (1977), } \\
\text { Gilmore y Pine (1997), De Dreu (1997) y Crittenden (1991). }\end{array}$} \\
\hline
\end{tabular}

Está claro que cuando una empresa se enfoca en un alto nivel de servicio al cliente, esto implica un nivel superior de costos para la empresa que no sucede cuando se enfoca en eficiencia y volumen. Cuando una empresa piensa que es una pérdida de oportunidad no dar respuesta tanto a salvaguardar los costos como ofrecer un nivel óptimo de servicio al cliente, es cuando surge lo que se denomina "personalización masiva" (Gilmore y Pine, 1997). Este concepto busca brindar, de manera masiva, los niveles de servicio/producto, acordes con los diferentes segmentos del mercado; así, se logra una preservación de los costes y un alto nivel de servicio al cliente.

Es necesario mencionar también la aparición de los conflictos a partir de las políticas y prácticas de los departamentos de recursos humanos, porque los elementos de medición, las expectativas en términos de competitividad, la asignación administrativa (autoridad, rol, responsabilidad, etc.), la eficiencia, la eficacia, entre otras, generan conflictos en cada departamento y a su vez, entre el individuo y su vida familiar (Kossek y Ozeki, 1998; Thompson, 1960).

\subsection{Interrelación entre los departamentos para manejar eficazmente el conflicto}

Existen ciertas interrelaciones entre los departamentos que ayudan en la administración de los conflictos. De Dreu y De Vliert (1997) afirman que el conflicto se debe mantener en un nivel óptimo y que, por tanto, los directivos deben seguirlo de cerca, observando la frecuencia con la que se está presentando y el nivel de su gravedad. Esta gravedad depende de su entorno y se da en varios niveles: se considera grave cuando sus características y costos reclaman mayor atención que cualquier otra preocupación; tanto asi que, si hubiere tal preocupación, esta pasará a un nivel muy secundario (Morales y Yubero, 1999). Entonces, la gravedad del conflicto estará dada por el impacto que tenga, o proyecte tener, en la organización. Un conflicto grave puede hacer caer una organización (Slipak y Hernández, 2003). Un conflicto si es bien manejado puede ser fuente de cambios y progresos interesantes para la compañía, La diferencia, el conflicto o el problema que no son tratados adecuadamente en la organización, afectan todo: baja la productividad y desanima (Barre$\mathrm{ra}, 2004)$.

Una forma de administrar el conflicto es apelando al plan de marketing, elemento estratégico que define algunos puntos de interrelación departamental; esto es, las $4 \mathrm{P}$ del marketing mix aplicadas: producto (o servicio), plaza (o distribución), precio y promoción (o comunicación) (Kotler, 1967). En otras palabras, es la introducción de estrategias que permiten articular una propuesta de entrega al cliente o consumidor, en la que participen todos los departamentos que tienen que ver con la misma, con el único fin de satisfacer plenamente al cliente.

Crittenden ${ }^{2}$, propone una nueva visión al respecto del plan de marketing, la cual implica las ${ }_{4} \mathrm{C}$ (centralizarse en el cliente, capacidades competitivas, colaboración, conexiones cíclicas). Así como se puede trabajar el conflicto para modificar y generar un marketing mix, también se puede tratar el conflicto enfocándolo en el cliente, es decir, manejando las conexiones cíclicas, la colaboración y las capacidades competitivas. En cuanto a las conexiones cíclicas que se generan cuando -a través de la implementación de la estrategia de marketing- se afecta la formulación de la misma, es necesario hacer una reformulación permanente, para que el conflicto sea una fuente útil en el mejoramiento permanente de la estrategia es decir, para satisfacer el cliente y los objetivos organizacionales (Crittenden, 2005), el proceso de 
colaboración generado debe darse dentro de un marco de referencia representado por uno o unos objetivos en común, sumado a las capacidades competitivas potencializadas por cada área. Todo ello debe estar enfocado en la visión de entrega de satisfacción y bienestar para el cliente y el cumplimiento de los objetivos de desempeño organizacional.

De esta manera, toda la organización buscará el logro de la meta común por encima de las metas internas de cada área. Según Crittenden, Gestalt explica muy bien la interacción de estos 8 axiomas del marketing, pues en esta teoría se puede cimentar el análisis del proceso de pedidos (4C), lógicamente, estudiando los comportamientos específicos del marketing (4P). Este enfoque tiene presente que el todo es mayor que la suma de sus partes (Crittenden, 2005). Shapiro (1988) está de acuerdo con la eficaz herramienta del marketing, pero dice que debe ser modificada y adaptada a los nuevos requerimientos de los mercados y de las organizaciones. Crittenden (1991) establece propuestas para el logro de una interrelación enfocada con el consumidor/cliente:

* Lo primero es centrarse en el cliente. El cliente externo es el receptor de los productos y/o servicios de la empresa y debe ser el enfoque central de la compañía; su satisfacción es crucial para el éxito. El cliente interno es la fuente del éxito, así como la satisfacción del cliente externo es el objetivo, el lograrlo depende del cliente interno ${ }^{3}$.

* Lo segundo es aprovechar las capacidades de cada departamento que puedan establecerse como ventajas competitivas para el logro de la satisfacción del cliente.

* Lo tercero es generar lazos fuertes de colaboración entre los departamentos, a través de metas y objetivos compartidos e interiorizados, incluso vinculando al cliente mismo al logro de los objetivos mediante procesos de co-creación.

* Y, en cuarto lugar, aprovechar el impacto que la implementación de la estrategia de marketing ocasiona en la formulación de la misma para generar un ciclo permanente de impacto-reformulación, aprovechando el conflicto que se genera para establecer un proceso de mejoramiento continuo de la estrategia de marketing.

Las compañías, por lo general, tratan de buscar cómo estar orientadas hacia el mercado, hacia sus clientes. Shapiro (1988) indica que las empresas pueden enfrentarse a diferentes dilemas cuando tratan de implementar un modelo orientado al mercado. Estar orientado al mercado implica que los procesos relacionados con el cliente y su satisfacción deben entrar en contacto con todas las áreas de la empresa. Es estar cerca del cliente. Así pues, se puede decir que hay tres características fundamentales que hacen que una empresa esté orientada al mercado:

* La información de todas las influencias de compra está en contacto con cada una de las funciones corporativas. En otras palabras, la empresa entiende su mercado y a las personas que deciden comprar sus productos o servicios. Como los clientes tienen diversas necesidades, es poco probable que se pueda satisfacer a un grupo amplio de estos. Se requerirá, entonces, de la colaboración entre las diferentes áreas con el fin de identificar aquellos clientes y segmentos del mercado que son claves.

* Las decisiones estratégicas y tácticas están hechas interfuncional e interdivisionalmente: cada área debe tener el punto de vista de las otras y deben estar dispuestas a escuchar, de manera activa, a las demás áreas. Con el fin de tomar decisiones acertadas, las áreas deben reconocer las diferencias que tienen entre sí. Porque la clave de estar orientado al mercado yace, en su mayoría, en la capacidad y la forma como las áreas negocian entre sí.

* Las áreas toman decisiones bien coordinadas y las ejecutan con un sentido de compromiso: un diálogo abierto en cuanto a las compensaciones es la mejor manera de lograr el compromiso hacia el logro de objetivos. De igual manera, cuando los que implementan son los mismos que planean, el compromiso es mucho mayor. Unas conexiones internas poderosas hacen que la comunicación sea clara, la coordinación sea fuerte y el compromiso alto.

\section{Aspectos metodológicos 3.1 Marco contextual}

La población objeto de estudio de esta investigación estuvo representada por diferentes personas vinculadas a distintas empresas del sector de la producción, que laboraban en los departamentos de marketing, producción o logística. Estas empresas -pequeñas, medianas y grandes- en su mayoría, están dedicadas a la manufactura de productos de consumo masivo y se encuentran localizadas en las ciudades de Cali y Bogotá.

\subsection{Herramientas utilizadas}

Metodológicamente, se procedió de esta manera: a) se revisó la literatura asociada con los conflictos inter e intradepartamentales en las organizaciones. b) Se

3. Schonberger (1990) afirma: "Servir al cliente externo es la 'gran meta', pero sólo puede ser obtenida de manera exitosa, si todos los clientes internos están comprometidos". 
seleccionó un conjunto de preguntas, cuya intención, más que medir, era la de descubrir las percepciones que los integrantes de cada uno de los departamentos de marketing, producción y logística tenían de sí mismos como departamento y de la labor de los otros dos departamentos. c) Se diseñó un cuestionario, tipo entrevista en profundidad, con preguntas tanto abiertas como acotadas. Este cuestionario se envió por correo electrónico a los profesionales laboralmente activos, de manera indiferenciada, en empresas pertenecientes al sector de consumo masivo y a los departamentos, o áreas de interés, con el fin de identificar fuentes y tipos de conflicto. Asimismo, se les pidió que compartieran el cuestionario con otras personas que tuvieran un perfil similar o igual al de ellos, es decir que estuvieran vinculadas en alguna de estas áreas en compañías del mismo sector. d) Por medio del análisis directo y documental, se establecieron los diferentes constructos para generar una herramienta de propuesta para investigaciones futuras.

En el cuestionario, se hicieron preguntas para establecer aspectos como la importancia relativa del área de desempeño frente a las otras dos, principios y valores del área, puntos de contacto con las otras dos áreas, conflictos percibidos en puntos de contacto, razones percibidas del conflicto, percepción de cómo afectan esos conflictos tanto interna como externamente (la repercusión hacia el mercado) y las acciones que debían hacerse para evitar los conflictos.

El análisis se llevó a cabo en 100 cuestionarios recopilados, a partir de los cuales, junto con la teoría, se concretaron los constructos, los cuales servirán de modelos para las futuras investigaciones que se hagan en las empresas colombianas acerca de los conflictos que surgen en estas áreas. Esta herramienta se aplicó en el personal, indistintamente de su cargo u profesión, pero con experiencia laboral activa en alguna de las tres áreas de interés: marketing, logística o producción, pertenecientes al sector de consumo masivo.

Entre las preguntas que se hicieron están: ¿Cuál es el objetivo u objetivos en el que más se esfuerza su área? ¿Cómo se percibe en su área, el esfuerzo realizado por las otras áreas? En esta misma investigación, se involucró la estrategia organizacional, para la cual se tomaron los mismos indicadores ya mencionados, pero con una diferencia en criterio: en lugar de eficiencia, se habló de precio. Así, se evaluó la percepción del entrevistado, con respecto a la estrategia organizacional. También se evaluó la presencia de conflictos y el nivel de gravedad de los mismos, instando, por medio de preguntas abiertas a sugerir situaciones reales y a calificar su importancia. Todos estos aspectos deberán ser tenidos en cuenta para futuras investigaciones que utilicen como base estos constructos aquí desarrollados.

\section{Resultados obtenidos}

4.1. Conflictos encontrados

En el desarrollo de la investigación, se encontraron los siguientes tipos de conflictos, presentados no solo dentro de cada área, sino también en las áreas propias de la investigación.

* Sensación de interdependencia entre las áreas.

* Problemas por no aprobación o retención de productos.

* Paro de equipos en producción por mantenimiento o por mal funcionamiento.

* Cambios en los programas de producción e incumplimiento de los mismos.

* Falta de materia prima.

* Cantidad de producción comprometida superior a la capacidad instalada (backorders).

* Problemas con pruebas y experimentos para prototipos y mejoras.

* Incumplimiento de normas de seguridad.

* Políticas de administración de personal.

* Mal manejo de materias primas, materiales y producto terminado.

* Desconocimiento de lo que hace la otra área.

* Desconocimiento de los factores técnicos y complejidad de lo que se produce.

* Falta de documentación.

* Falta de proactividad.

* Falta de conocimiento de la competencia y del consumidor.

* Incoherencia en los objetivos propios de cada área.

* Las áreas se culpan de no cumplir con su respectiva labor.

* Falta de personal idóneo en las demás áreas.

* Actitud negativa.

4.2. Generación de constructos para la investigación de los conflictos interdepartamentales entre marketing, producción y logística

A continuación, se exponen los 8 constructos que surgieron a partir de la revisión teórica y del contraste con los resultados de los 100 cuestionarios que se aplicaron. Cada constructo se puede medir a partir de los elementos que los describen. 


\begin{tabular}{|c|c|}
\hline Constructo & Elementos que lo describen \\
\hline $\begin{array}{l}\text { Satisfacción del } \\
\text { cliente }\end{array}$ & $\begin{array}{l}\text { * Disponibilidad de producto } \\
\text { * Entrega a tiempo } \\
\text { * Precio } \\
\text { * Soporte técnico } \\
\text { * Amplitud de línea } \\
\text { * Calidad técnica } \\
\text { * Confiabilidad } \\
\text { * Diseño }\end{array}$ \\
\hline $\begin{array}{l}\text { Conectividad } \\
\text { interdepartamental }\end{array}$ & $\begin{array}{l}\text { * Facilidad de comunicación } \\
\text { * Facilidad de acceso interdepartamental }\end{array}$ \\
\hline $\begin{array}{l}\text { Conflicto } \\
\text { interdepartamental }\end{array}$ & $\begin{array}{l}\text { * Nivel y tipo de relaciones entre departamentos } \\
\text { * Frecuencia de la aparición de conflictos entre } \\
\text { los departamentos } \\
\text { * Sensación de uniformidad de objetivos } \\
\text { * Compatibilidad de objetivos entre } \\
\text { departamentos }\end{array}$ \\
\hline $\begin{array}{l}\text { Coordinación } \\
\text { interdepartamental }\end{array}$ & $\begin{array}{l}\text { * Los departamentos comparten información } \\
\text { * Los departamentos tienen la estrategia } \\
\text { integrada } \\
\text { * Los departamentos contribuyen con el valor a } \\
\text { cliente }\end{array}$ \\
\hline $\begin{array}{l}\text { Factores } \\
\text { demográficos }\end{array}$ & $\begin{array}{l}\text { * Edad } \\
\text { * Años de experiencia } \\
\text { * Área de experiencia } \\
\text { * Experiencia en otras áreas } \\
\text { * Sector de la industria } \\
\text { * Estado civil } \\
\text { * Composición familiar }\end{array}$ \\
\hline $\begin{array}{l}\text { Variables de } \\
\text { relación }\end{array}$ & $\begin{array}{l}\text { * Nivel de importancia por el trabajo conjunto } \\
\text { entre las áreas, para identificar necesidades de } \\
\text { clientes } \\
\text { * Nivel de importancia por el trabajo conjunto } \\
\text { entre las áreas, para satisfacer las necesidades } \\
\text { de los clientes } \\
\text { * Nivel de importancia por la creación de } \\
\text { grupos de trabajo interfuncionales } \\
\text { * Nivel de importancia por el conocimiento de } \\
\text { las otras áreas } \\
\text { * Nivel de importancia por el status } \\
\text { organizacional de las áreas } \\
\text { * Nivel de importancia por la integración de las } \\
\text { áreas } \\
\text { * Nivel de importancia por el entrenamiento } \\
\text { interfuncional }\end{array}$ \\
\hline $\begin{array}{l}\text { Factores de } \\
\text { medición (es } \\
\text { necesario } \\
\text { desarrollar cada uno } \\
\text { de ellos, de acuerdo } \\
\text { con la población que } \\
\text { se va a estudiar, } \\
\text { cuando se apliquen } \\
\text { en la investigación) }\end{array}$ & $\begin{array}{l}\text { * Nivel de relación } \\
\text { * Trabajo en equipo } \\
\text { * Nivel de conflicto } \\
\text { * Conocimiento } \\
\text { * Nivel de integración } \\
\text { * Calidad y cantidad de contactos entre las áreas }\end{array}$ \\
\hline Fuentes de conflicto & $\begin{array}{l}\text { * Educación y entrenamiento } \\
\text { * Entendimiento } \\
\text { * Objetivos y prioridades } \\
\text { * Restricciones de costo y tiempo } \\
\text { * Comunicaciones } \\
\text { * Internas en cada departamento } \\
\text { * Sentimientos personales } \\
\text { * Desconfianza } \\
\text { * Administración } \\
\text { *Cambios organizacionales } \\
\text { * Políticas de empresa: recursos humanos, } \\
\text { interdepartamentales, departamentales }\end{array}$ \\
\hline & Fuente: Varón, A. (2013) \\
\hline
\end{tabular}

Todos estos constructos deben ser definidos en los términos (significados y significantes) de la población objeto de investigación o estudio.

\section{Conclusiones}

Los conflictos se presentan regularmente en los entornos laborales y pueden ser positivos y negativos. Muchas de las investigaciones previas muestran que los administradores que entendían la lógica de los conflictos rechazaban el componente emocional inherente al conflicto y no aceptaban que afectaba el proceso de toma de decisiones. De ahí, la importancia de solucionarlos.

Al revisar detenidamente la bibliografía y los estudios realizados en este campo, se observó que existen modelos empíricos para el manejo de conflictos en general. Pero no existe uno que vincule las áreas de marketing, producción y logística con la satisfacción del consumidor.

Aunque las empresas han aplicado modelos de integración, los conflictos se siguen presentando entre estas tres áreas, cada vez con mayor frecuencia. Estos conflictos están comúnmente relacionados con los objetivos y valores propios de cada área. De hecho, se encontraron sentimientos de insatisfacción de los miembros de cada área con respecto a la labor de las otras dos. Frente a este panorama, el constructo teórico propuesto dará un soporte para abordar el tema en una investigación futura.

\section{Recomendaciones}

Para que el tan anhelado éxito organizacional y la prosperidad de la compañía, sea posible, es necesario que todas sus áreas operen en una atmósfera de cooperación y coordinación de tareas, que llevará a la plena satisfacción del consumidor.

A los estudiosos sobre este tema, se les invita a diseñar modelos prácticos para el manejo de los conflictos empresariales, donde se vinculen las áreas de marketing, producción y logística con la satisfacción del consumidor.

Estos constructos se pueden usar para investigar los posibles conflictos entre los departamento de marketing, producción y logística; se aconseja que se desarrollen con escalas de medición tipo Likert, de $7 \mathrm{u}$ 11 puntos. Así, se generan valores que, luego permitirán establecer formulaciones matemáticas o relacionales para la generación de un modelo causal.

Como los conflictos están relacionados con los objetivos y valores propios de cada área, es pertinente que los directivos de marketing, producción y logística trabajen de manera mancomunada, para que sus funcionarios logren unir adecuadamente los objetivos y valores de estas tres áreas; así se podrá superar cualquier inconveniente que los lleve a un conflicto sin solución. 


\section{Referencias}

Aktouf, 0. (1990). Le Symbolisme Et La «Culture D'entreprise». Des Abus Conceptuels Aux Leçons Du Terrain. En: Chanlat, J.F. L'individu Dans L'organisation: Les Dimensions Oubliées. Québec, Canadá: Pul/ESKA.

Aktouf, O. Cruz Kronfly, F. y Carvajal, R. (2003) El lado inhumano en las organizaciones. Cali, Colombia: Facultad de Ciencias de la Administración - Universidad del Valle.

Amason, A. (1995). Conflict an important dimension in successful management. Teams. Organizational Dynamics, 24 (20-34).

Baron, R. (1991). Positive effects of conflict. Employee Responsibilities and Rights, 4 (pp. 25-36).

Barrera-Santos, R.Y. (2004). El conflicto organizacional. En: Barrera Santos, R.Y. Negociación y transformación de conflictos: reto entre escasez y bienestar. ( $2^{\text {da }}$ ed. Ampliada). Ciudad de Guatemala, Guatemala: Editorial Serviprensa (pp. 43-64)

Bazaraa, M. \& Jarvis, J. (1977). Linear programming and network flows. New York, USA: Wiley.

Bourgeois, L. (1985). Strategic goals, percieved uncertainty, and economic performance in volatile environments. Academic Management, 28 (pp. 548-573).

Brewer, M. (1986). Choice behavior in social dilemmas: effects of social identity, group size and decision framing. Journal of Personality and Social Psychology, 3 (pp. 543-549).

Brewer, M. \& Schneider, S. (1990). Social identity and social dilemmas: a double-edged sword. In: Abrams, D. \& Hogg, M.A. (Eds.). Social identity theory: constructive and critical advances. London, UK, New York, USA: Harvester Wheatsheaf.

Brown, L. (1983). Managing conflict at organizational interfaces. Reading, USA: Addison Wesley.

Burns, T. \& Stalker, G. (1966). The management of innovation ( $2^{\mathrm{a}}$ ed.). London, UK: Thavistock.

Crittenden, V. (1991). Crossing functional lines: achieving successful strategy implementation through effective internal partnering. Industrial Management, 33 (1) (pp. 26-28). . (1992). Close the marketing/manufacturing gap. Sloan Management Review, 33 (3) (pp. 41-52).

_. (1993). Reducing conflict between marketing and manufacturing. Industrial Marketing Management, 22 (pp. 1-11). . (2005). The rebuilt marketing machine. Business Horizons, 48 (pp. 409-420).

Dahler-Larsen, P. (1998). What 18 case studies of organizational culture tell us about counter-intentional effects of attempts to establish shared values in organizations. In: Rahim, M.A. Golembiewski, R.T. \& Lundberg, C.C. (Eds.). Current Topics in Management (Vol. 3). Stanford, USA: Jai Press (pp. 151173).

De Dreu Carsten, K.Y. \& De Vliert Van, E. (1997). Using conflict in organizations. London, UK: Sage ed.

Dyer, B. \& Song, M. (1997). The impact of strategy on conflict: a cross-national comparative study of U.S. and Japanese firms. Journal of International Business Studies, 28 (3) (pp. 467-493).

Esquivel, M. (1997). The importance of conflict in work team effectiveness. Team Performance Management Bradford, 3 (2) (pp. 89-96).

Galbraith, J.R. (1973). Designing complex organization. Reading, USA: Addison-Wesley
Gilmore, J. \& Pine, B. (1997). The four faces of mass customization. Harvard Business Review, 75 (1) (pp. 91-101).

Goldratt, E. y Cox, J. (1993). La meta: un proceso de mejora continua. Monterrey, México: Ediciones Castillo.

Hayes, R. \& Wheelwright, S. (1984). Restoring our competitive edge. New York, USA: John Wiley,

Higgins, J. (1991). The management challenge ( $\left.2^{\text {nd }} E d\right)$. Boston, USA: Macmillan Publishing.

Kamarkar, U. (1996). Integrative research in marketing and operations management. Journal of Marketing Research, 33 (pp. 125-133).

Kotler, P. (1967). Marketing management: analysis, planning, and control. Englewood Cliffs, USA: Prentice Hall

Kossek, E. \& Ozeki, C. (1998). Work-family conflict, policies, and the job-life satisfaction relationship: A review and directions for organizational behavior-human resources research. Journal of Applied Psychology, 83 (2) (pp. 139-149)

Laurence, P. \& Lorsch, J. (1967). Organization and environment. Homewood. USA: Richard D. Irwin, Inc.

Miller, J. \& Roth, A. (1994). A taxonomy of manufacturing strategies. Management Science 40 (3) (pp. 285-304).

Mintzberg, H. (1979). The structuring of organization. Englewood Cliffs, USA: Prentice-Hall.

Morales, J.F. y Yubero, S. (1999). El grupo y sus conflictos. Cuenca. España: Ediciones de la Universidad Castilla la Mancha.

Nauta, A. (2001). Causes and consequences of perceived goal differences between departments within manufacturing organizations. Journal of Occupational and Organizational Psychology, 74 (pp. 321-342).

Ouchi, W. (1980). Markets, bureaucracies and clans. Administrative Science Quaterly, 25 (pp. 129-141).

Pondy, L.R. (1966). A systems theory of organizational conflict. Academy of Management Journal, 9 (3) (pp. 246-256).

Porter, M. (1980). Competitive strategy: techniques for analyzing industries and competitors. New York, USA: Free Press.

Powers, T.L., Sterling, J.U. \& Wolter, J.F. (1988), Marketing and manufacturing conflict: sources and resolution. Production and Inventory Management Journal, 1 (1) (pp. 56 - 6o).

Prabhaker, P. \& Goldhar, J (1995). Marketing implications of newer manufacturing technologies. Journal of Business \& Industrial Marketing, 10 (2) (pp. 48-58).

Putnam, L. (1994). Productive conflict: negotiation as implicit coordination. Conflict Management, 5 (pp. 284-298).

Roethlisberger, F. (1965). Management and morale. Cambridge, UK: Harvard University Press.

Sampietro, D. (20/03/2006). Evolución del Trade Marketing y su relación con Category Management. Revista Amai (pp. 36 41). Recuperado 01/05/07 de: http://www.amai.org/pdfs/ revista-amai/revista-amaiarticulo-20060320_120532.pdf

Schonberger, R. (1990). Building a chain of customers. New York, USA: Free Press.

Schwenk, C. \& Cosier, R. (1993). Effects of consensus and devil's advocacy on strategic decision making. Journal of Applied Social Psychology, 23 (2) (pp. 126-139).

Shapiro, B. (1977/09/00). Can marketing and manufacturing coexist? Harvard Business Review, (pp 104-112). (1988/11/00). What the hell is market oriented? Harvard Business Review, (pp 1-7).

Slipak, O.E. y Hernández, M. (2003). Estrés laboral. Psicología y Psiquiatría. Antropología y Sociología. Recuperado 03/o8/2011 de: http://mobbingopinion.bpweb.net/artman/ publish/printer_3864.shtml 
Thamhain, H. \& Wilemon, D., (1975). Diagnosing conflict determinants in Project management. Engineering Management, 22 (pp. 35-44).

Thomas, K.W. (1992). Conflict and negotiation processes in organizations. In: Dunnette, M.D. \& Hough, L.M. (Eds.), Handbook of industrial \& organizational psychology ( $2^{\text {nd }}$ ed., Vol. 3). Palo Alto, USA: Consulting Psychologists Press (pp. 652-717).

Thompson, J.D. (1960). Organizational management of conflict. Administrative Science Quarterly, 4 (4) (pp. 389-409).

Walton, R. \& Lawrence, P.(1985). Human resource management trends and challenge. Cambridge, USA: Harvard Business School Press.
Whetten, D. \& Cameron, K. (1995). Developing management skills, ( $3^{\text {rd }}$ ed.). New York, USA: Harpercollins.

$\mathrm{Xie}$, J. (1998). Interfunctional conflict, conflict resolution styles, and new product success: a four - culture comparison. Management Science, 44 (12) (pp. 192- 206).

Zapata D,Á. y Rodríguez R,A. (2008). Componentes, rasgos y características de la cultura en el análisis organizacional. En: Zapata D.A. y Rodríguez R.A. (Eds). Gestión de la cultura organizacional. Bases conceptuales para su implementación. Cali, Colombia: Facultad de Ciencias de la Administración - Universidad del Valle.

Cuadernos de Administración / Facultad de Ciencias de la Administración / Universidad del Valle Periodicidad: semestral / ISSN impreso N 0120-4645- ISSN electrónico N 2256-5078 / Nombre abreviado: cuad.adm. Edición Vol. $29 \mathrm{~N}^{\circ} 49$ (enero - junio de 2013)

Constructos teóricos para abordar, de un modo investigativo, problemas entre marketing, producción y logística en las empresas colombianas / Alexander Varón Sandoval 